\title{
Viewpoint-invariance of contour-curvature discrimination over extended performance levels
}

\author{
DAVID H. FOSTER* and DAVID R. SIMMONS $\dagger$ \\ Department of Communication and Neuroscience, Keele University, Staffordshire ST5 5BG, UK
}

Received for publication 11 October 1993

\begin{abstract}
A traditional approach to the problem of human object recognition is to assume that the visual system represents an object in terms of invariant quantities. This study considers a generalization of this approach to the problem of visually recognizing differences in shape, specifically between contour markings on a planar surface, as the position of the surface varies in space. For a given 'shape cue', perceived differences in contour may be quantified by threshold values-Weber fractions-at a particular criterion level of performance. A necessary theoretical condition for the Weber fraction to be constant with the relative viewpoint of the observer is that the cue should be a relative invariant under the natural spatial transformations of the image. Some data are reviewed showing how statistically efficient some cues are in explaining the observed discriminability of symmetric curved contours at a fixed criterion performance level of $75 \%$. A new, fuller analysis is presented showing that the efficiency of the most efficient cue satisfying the invariance condition is maintained over a wide range of criterion performance levels, from $53 \%$ to $92 \%$ correct, corresponding to discrimination index values of 0.1 to 2.0. Over these levels, Weber's law was found empirically to hold almost exactly.
\end{abstract}

\section{INTRODUCTION}

The problem of object or pattern recognition-at one time known as the problem of 'stimulus equivalence'-has been summarized generally by Dodwell (1992) thus:

How can a pattern be recognized as such (e.g. a melody, a face) despite the fact that it can occur at different pitches, tempi or timbres in the one case, different sizes, orientations and colours in the other? (p. 511 )

One long-standing approach to this problem, investigated by Dodwell in a wide range of studies of human and animal vision (Dodwell, 1957, 1970, 1992), is to consider it in terms of the encoding process (Dodwell, 1992; see also Foster, 1977). According to this approach, recognition is achieved, despite changes in the position and orientation of the object and of the observer, by the visual system's forming a representation of the object-or parts of it-in terms of quantities that are stable under those changes. These quantities are the invariants of the natural spatial transformations involved: translations and rotations with respect to the three Cartesian axes. An example of such an invariant quantity is the cross-ratio for four points on a line: the ratio of the ratios of the distances between the points remains constant as the line is translated and rotated in space (see e.g. Mundy and Zisserman, 1992; Reiss, 1993). Psychophysical measurements involving the cross-ratio have been reported by Cutting (1986) and Niall (1992).

\footnotetext{
*To whom correspondence should be addressed.

$\uparrow$ Present address: McGill Vision Research, Department of Ophthalmology, McGill University, 687

Pine Avenue West, H4-14, Montréal, Québec H3A 1 Al, Canada.
} 
This study considers a generalization of this invariance-based approach to a complementary question. Suppose that there is on the surface of an object a pattern of contours formed by markings due to sharp changes in surface reflectance, and not to self-occlusion or shadow. Can the differences in shape between these contours be recognized despite changes in the viewing distance and direction of the observer? In practice, judgements about differences in shape may be just as important as judgements about identities in shape; as with identification, it may be desirable that judgements about differences are, as far as possible, invariant with changes in relative viewpoint. In short, the differences between the parts of a surface should be perceived to be a property of the object, not of the viewpoint of the observer. ${ }^{1}$ An example of where it is essential that contour shape differences are attributed to the object rather than to the viewpoint is in the interpretation of surface three-dimensional structure from surface contours (Knill, 1992). In practice, discrimination may fail to be viewpoint-invariant through causes unrelated to the invariances themselves; for example, occlusion of the surface (self-occlusion and by other objects), and image scale (the image may be so small that it is not resolved or so large that it passes outside the visual field). These factors are excluded from this analysis.

A central question for object discrimination (and for object identification) is the nature of the quantity used to make these perceptual judgements; that is, the 'shape cue'. The following sections consider in turn: (1) how, for a given cue, perceptual differences in shape may be quantified in terms of thresholds-Weber fractions-obtained at a particular criterion level of discrimination performance; (2) how, theoretically, the invariance of the Weber fraction under changes in the relative viewpoint of the observer is related to the relative invariance of the cue under spatial transformations of the image; (3) how well some cues (relative invariant or not) explain the observed discriminability of symmetric curved contours for a particular criterion performance level; and (4) how well such an explanation by one relative-invariant cue holds for all reasonable criterion performance levels.

This study is concerned only with what is sometimes called geometric invariance; it is not concerned with the effects of change in illumination (photometric invariance), or with the effects of visual context or occlusion. Planar shapes only are considered. Although the theory is-within these constraints-general, applications are based on the task of discriminating curvedline fragments in the plane. Contour curvature is an important local determinant of perceived planar shape (Attneave, 1954; Koenderink and van Doorn, 1982; Richards et al., 1986; Link and Zucker, 1988).

\section{QUANTIFYING PERCEPTUAL DIFFERENCES}

Suppose that some physical shape attribute is chosen and that it has values $c$ on some continuum. Visual sensitivity to differences in shape may be summarized by the size of the smallest detectable difference $\Delta c=c^{\prime}-c$ between values $c, c^{\prime}$, at some criterion level of performance; for example, per cent correct in a forcedchoice task (shapes with attributable values $c, c^{\prime}$ are discriminable on $p \%$, say, of occasions, and not discriminable on $(100-p) \%$ of occasions). The quantity $\Delta c$ is the increment or difference threshold (Falmagne, 1985; Laming, 1986), and 
the ratio $\Delta c / c$ is the Weber fraction. Weber's law (Weber, 1834), in its general form, asserts that $\Delta c$ depends linearly on $c$; that is, the Weber fraction is constant. Clearly, Weber's law need not hold for all shape attributes.

For curved contours, there are many shape attributes that could be used to quantify discrimination performance. Eight candidate cues of a mainly geometric kind are as follows: Euclidean curvature (or, if the curved contour is not circular, an 'equivalent curvature' defined for a circular arc with the same endpoints and midpoint as the fragment ${ }^{2}$ ); radius of (equivalent) curvature; the angle turned through by a tangent moving along the curve; the maximum deviation of the curve from linearity ('sag'); the mean deviation from linearity; the area enclosed by the curve, the arc-length of the curve; and the quotient of arc-length by chord-length (for details, see Foster et al., 1993a; Foster and Wagemans, 1993). Which of these cues satisfies the viewpoint-invariance condition introduced in Sect. 1 is considered in the next section. The question of empirical acceptability is addressed later.

\section{DISCRIMINATION CUES AND VIEWPOINT INVARIANCE}

The requirement that judgements about differences in planar shape should, as far as possible, be invariant with changes in viewpoint can be expressed by saying that the Weber fraction should be viewpoint invariant. As noted earlier, the Weber fraction is specified for a particular criterion level of performance. For such a level, the viewpoint-invariance condition implies that the ratio $c^{\prime} / c$, where $c^{\prime}=$ $c+\Delta c$, should be constant. This requirement-that the ratio of values be constant-is equivalent to saying that the cue itself should be a relative invariant under the natural spatial transformations of the object, or under the corresponding natural spatial transformations of the image, which are defined explicitly in this section. A relative invariant is the same as an (absolute) invariant except that values of the invariant are scaled by a number that depends on the 'size' of the transformation (Mundy and Zisserman, 1992).

The eight aforementioned geometric attributes of curved-line fragments have been analysed previously by algebraic methods (Foster and Wagemans, 1993) for invariance of an arbitrary ratio $c^{\prime} / c$ under natural spatial transformations of the image. These changes in relative viewpoint may be represented by affine transformations of the fronto-parallel image plane (affine transformations map parallel lines onto parallel lines), providing that the portion of the object plane containing the contours is so far from the eye that the depth of the contours is relatively small; that is, perspective effects may be ignored (see Aloimonos, 1990; Mundy and Zisserman, 1992; Reiss, 1993).

Since measurements of curved-line discriminability have traditionally used stimuli with one symmetry axis and parallel chords (Foster and Wagemans, 1993; see Fig. 1a), the affine transformations were assumed to be symmetry-preserving; in the usual Cartesian coordinates (see Fig. 1b), the transformations were therefore of the form:

$$
T:(x, y) \rightarrow(a x, b y),
$$

where the scale factors $a, b \geq 0$. These transformations actually consisted of a combination of enlargement (or reduction) in the $x$-and $y$-directions $(x, y) \rightarrow$ 
(a)

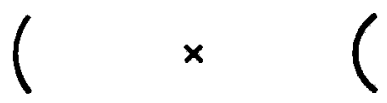

(b)

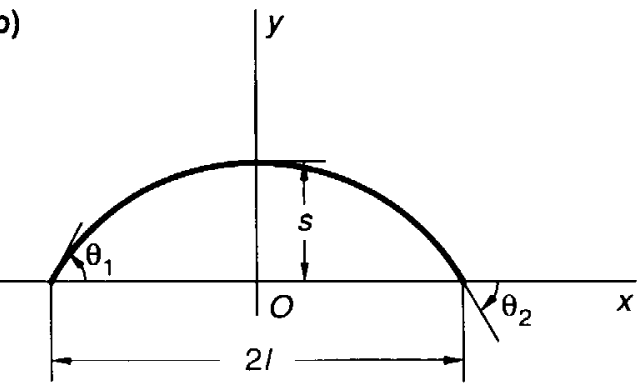

Figure 1. (a) Display configuration. Stimulus eccentricities were $1.4 \mathrm{deg}$ visual angle (measured to the midpoint of each chord); chord-lengths ranged from 0.2 to $0.8 \mathrm{deg}$. (b) Geometry of curved-line stimulus with chord-length $2 l$ and $\operatorname{sag} s$ (in the text, the symbol $c$ is used generally to include sag). The turning angle of the curved line is $\theta_{2}-\theta_{1}$. The equivalent-curvature of the curved line is the Euclidean curvature of a circular arc with the same endpoints and midpoint; equivalent-curvature is thus $2 \mathrm{~s} /\left(l^{2}\right.$ $+s^{2}$ ) (the Chord Theorem). Full computational formulae for these and other attributes, namely, arclength, arc-length-divided-by-chord-length, mean-deviation, and area, are given in Foster et al. (1993a) and Foster and Wagemans (1993).

$(a x$, ay), where $a>0$, and a simple elongation in the $x$-direction alone $(x, y) \rightarrow$ $(a x, y)$, where $a \geq 1$, or a simple compression in the $y$-direction alone $(x, y) \rightarrow$ $(x, a y)$, where $0 \leq a \leq 1$ (Foster et al., 1993a).

Informally, if the stimuli are imagined as arising from some suitably aligned physical markings on the planar surface of an object, the transformations $T$ would thus correspond to a combination of a shift in the object plane away from or towards the eye and a rotation of the plane about a vertical axis or about a horizontal axis; or to a complementary shift in the position of the observer.

With an abuse of notation, let $T(c)$ represent the value $c$ of the cue after $T$ has been applied to a curved-line fragment. Of the attributes listed in Sect. 2, those that did not yield constant ratios $T\left(c^{\prime}\right) / T(c)$ were equivalent-curvature, ${ }^{3}$ radiusof-equivalent-curvature, turning-angle, arc-length, and arc-length-divided-bychord-length; the attributes that did were sag, mean-deviation, and area (Foster and Wagemans, 1993).

This property of relative invariance, although motivated by the general arguments set out earlier, serves only to classify potential cues for shape discrimination into those which theoretically could allow the Weber fraction to be preserved and those which could not. The next section considers the relationship between this property and how cues might be assessed experimentally as to their appropriateness, without reference to Weber's law.

\section{CUE EFFICIENCY AT A SINGLE CRITERION LEVEL}

In the sense that a cue can be treated as a parameter of the stimulus, the problem of deciding its acceptability can be approached from the standpoint of statistical estimation theory (Fisher, 1922; Stuart and Ord, 1991). The technique (Foster et 
al., 1993a) is to determine which of a set of candidate cues gives the best prediction of measured increment threshold $\Delta c$ as a function of $c$; the 'best' predictor in the sense of statistical estimation theory being the most efficient one; that is, the one yielding the smallest variance in the data about the sample mean at each value of $c$. This notion of efficiency is distinct from the notion of efficiency defined with respect to a particular visual detector mechanism (Watt and Andrews, 1982).

Data for increment-threshold functions for contour-curvature discrimination were obtained in a series of experiments (Foster et al., 1993a). These experiments required the (forced-choice) discrimination of two simultaneously presented, 1-s duration, curved-line fragments (forming sections of ellipses), with parallel chords, whose curvatures varied from 0 to $0.13 \mathrm{arcmin}^{-1}$ and chordlengths from 12 to 48 arcmin (units of visual angle). The turning angle of the most curved curve was $120 \mathrm{deg}$. Figure 1a shows the experimental arrangement of the curved lines. For each candidate cue, increment threshold $\Delta c$ was obtained as a function of $c$ under combinations of enlargements or reductions and simple elongations or compressions of the curved lines, according to Eqn (1). The value of $\Delta c$ at each $c$ was derived from a psychometric function for each attribute and each observer. Figure 2 shows example increment-threshold data for sag and the fitted function; per cent correct discrimination ('which curved-line fragment was the more curved?') is plotted against the stimulus level, specified by the difference in sag value between one fragment and the other, with the smaller of the sag values being fixed, here at 2.57 arcmin. The smooth curve is a quadratic function of stimulus level, transformed by the standardized normal integral, and fitted by a maximum-likelihood procedure (GLIM, NAG, 1987). Each set of incrementthreshold data was fitted individually; a standard shape of psychometric function was not assumed, but the general quadratic form with three coefficients gave satisfactory fits under all conditions (Foster et al., 1993a). The criterion level of

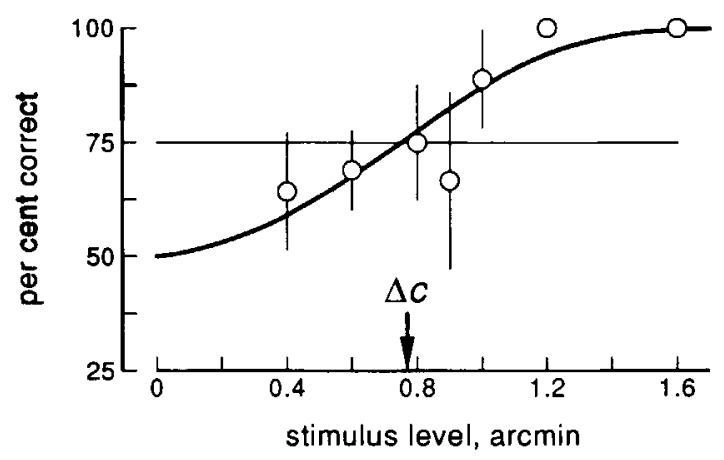

Figure 2. An example psychometric function for one subject and one experimental condition (chordlength $0.4 \mathrm{deg}$; one curved line of constant sag $2.57 \mathrm{arcmin}$ ). Per cent correct discriminations between the two curved lines ('which curved-line fragment was the more curved?') is plotted against stimulus level (the difference in sag value between one fragment and the other). The vertical bars are binomial estimates of the standard deviations associated with each data point (since the number of trials at each level was determined by an adaptive procedure, the sizes of the error bars were not necessarily the same at similar per cent correct values). The smooth curve is a quadratic function of stimulus level, transformed by the standardized normal integral, and fitted by a maximum-likelihood procedure. The criterion level of performance is $75 \%$, and the estimated increment threshold $\Delta c$ is shown arrowed. 
performance was set at $75 \%$, midway between chance level $(50 \%)$ and perfect performance $(100 \%)$. The estimated increment threshold $\Delta c$ for the data in Fig. 2 is shown arrowed.

Figure 3 shows two of the resulting increment-threshold functions (adapted from Foster et al., 1993a, Fig. 2) for equivalent-curvature and sag. Estimates of $\Delta c$ (averaged over five observers) are plotted as a function of $c$; the different symbols correspond to different sizes (chord-lengths) of the curved lines, as indicated in the legend to the figure. The broken lines are least-squares regressions, not including data at zero equivalent-curvature and sag owing to the possible influence of a separate discriminatory mechanism (see Foster et al, 1993a; Kramer and Fahle, 1993), but it is evident that no continuous curve could account adequately for the variance in the data for equivalent-curvature. ${ }^{4}$ Recall that the variance in the observed increment-threshold values is defined with respect to the sample mean at each value of $c$; for the variance to be minimum it is not necessary that the sample mean should vary linearly with $c$, or, equivalently, that Weber's law should hold.

In fact, none of the potential cues that failed the viewpoint-invariance condition, namely, radius-of-equivalent-curvature, turning-angle, arc-length, arc-length-divided-by-chord-length, and equivalent-curvature, accounted adequately for the variance in the data; and, of those that did satisfy the viewpoint-invariance condition, namely sag, mean-deviation, and area, the best predictor over all conditions was sag (Foster et al., 1993a).

\section{CUE EFFICIENCY AT MULTIPLE CRITERION LEVELS}

The experimental tests of cue efficiency summarized in the preceding section were performed at one criterion level of discrimination performance: $75 \%$ correct. If other levels had been chosen, would the efficiency of the most efficient cue have been maintained?

The resolution of this question is slightly complicated by the imprecision of the psychometric function. The stimulus testing levels associated with each function
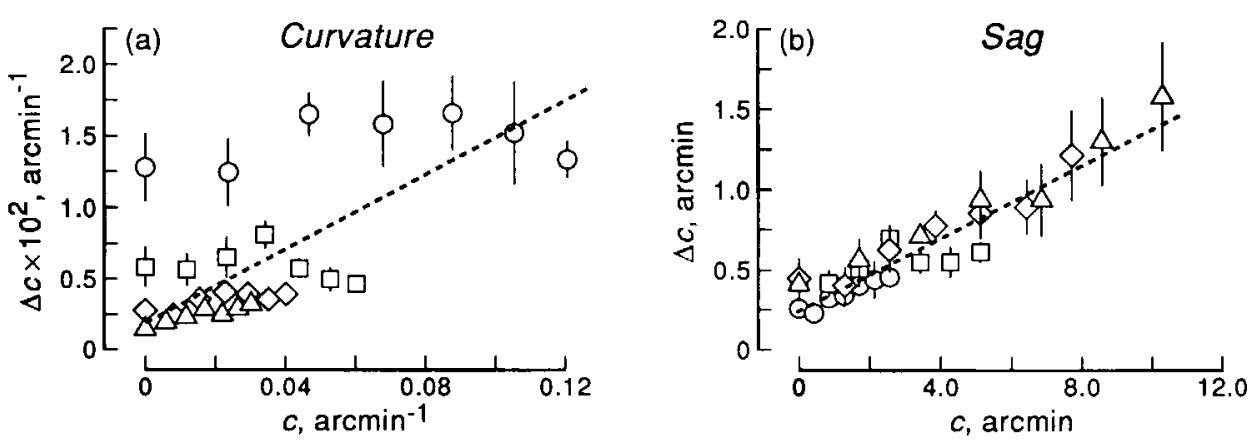

Figure 3. Increment-threshold functions for equivalent-curvature and sag. Estimates of increment threshold $\Delta c$ are plotted aginst reference value $c$ for four enlargements of the curved lines, specified by their chord-lengths: $\bigcirc 0.2 \mathrm{deg} ; \square 0.4 \mathrm{deg} ; \diamond 0.6 \mathrm{deg}$; and $\triangle 0.8 \mathrm{deg}$. Each data point is the weighted mean over five subjects, and the vertical bars show \pm 1 SEM. Broken lines are least-squares linear regressions (with the point $c=0$ arcmin omitted). Adapted from Foster et al., 1993a, Fig. 2, with kind permission from Pergamon Press Ltd, Headington Hill Hall, Oxford OX3 0BW, UK. 
(Fig. 2) were chosen in the original experiments (Foster et al., 1993a) according to a sequential adaptive testing algorithm (PEST, Taylor and Creelman, 1967; modified by Hall, 1981) that centred performance on the $75 \%$ correct criterion level; for other more extreme levels, the form of the fitted curve was theoretically less reliable, and consequently the thresholds derived from it. In the analysis of the raw data performed here, the uncertainty at each criterion level was quantified by a bootstrap estimate (Efron, 1982; Foster and Bischof, 1991) of the standard deviation of the corresponding threshold $\Delta c$. Weighted mean values of $\Delta c$ were then computed over observers. The criterion levels were chosen with reference to values of the discrimination index $d^{\prime}$ from signal-detection theory (Green and Swets, 1966): selected $d^{\prime}$ values were $0.1,0.2,0.5,1.0,1.5$, and 2.0 , corresponding to values of $53 \%, 56 \%, 64 \%, 76 \%, 86 \%$, and $92 \%$ correct, respectively.

Figure 4 shows estimates of sag increment threshold $\Delta c$ plotted as a function of sag $c$ at each of these criterion levels. As in Fig. 3, the different symbols correspond to different enlargements of the curved lines, and the broken lines are least-squares regressions (not including data at zero sag for the reason mentioned earlier). Table 1 summarizes the results of a formal analysis of variance with repeated measures (Winer, 1971, sections 4.2,4.6) using weighted values of sag increment threshold and without omission of the data at zero sag. The lack of linear fit was nowhere statistically significant, although at the most extreme criterion level there was an increase in the lack of fit that approached significance $\left(p=0.07\right.$ for $d^{\prime}=2.0$ )

Table 1.

Consistency of curved-line increment-threshold data (Fig. 4) with Weber's law at six criterion levels of discrimination performance. The $F$-ratios measure the lack of fit of linear functions of sag $c$ fitted to observed estimates of sag increment threshold $\Delta c$ at each $d^{\prime}$ criterion value.

\begin{tabular}{lll}
\hline Criterion level, $d^{\prime}$ & $\begin{array}{l}\text { Lack of linear fit } \\
F(26,108)\end{array}$ & $p$-value \\
\hline 0.1 & 1.37 & 0.13 \\
0.2 & 1.41 & 0.11 \\
0.5 & 1.02 & 0.45 \\
1.0 & 0.73 & 0.82 \\
1.5 & 0.98 & 0.51 \\
2.0 & 1.51 & 0.07 \\
\hline
\end{tabular}

\section{COMMENT}

The concern of this study has been with the problem of recognizing differences in planar shape as the relative viewpoint of the observer alters. Given a contourcurvature attribute with values $c$ on some continuum, how do threshold differences $\Delta c$ in $c$ vary with $c$ under natural image transformations, specifically under affine transformations preserving symmetry? One of the attributes considered, namely sag, has four important properties: (1) For curved lines with parallel chords, it is a relative invariant with respect to affine transformations, a theoretical condition for preserving the Weber fraction. (2) At the criterion level 

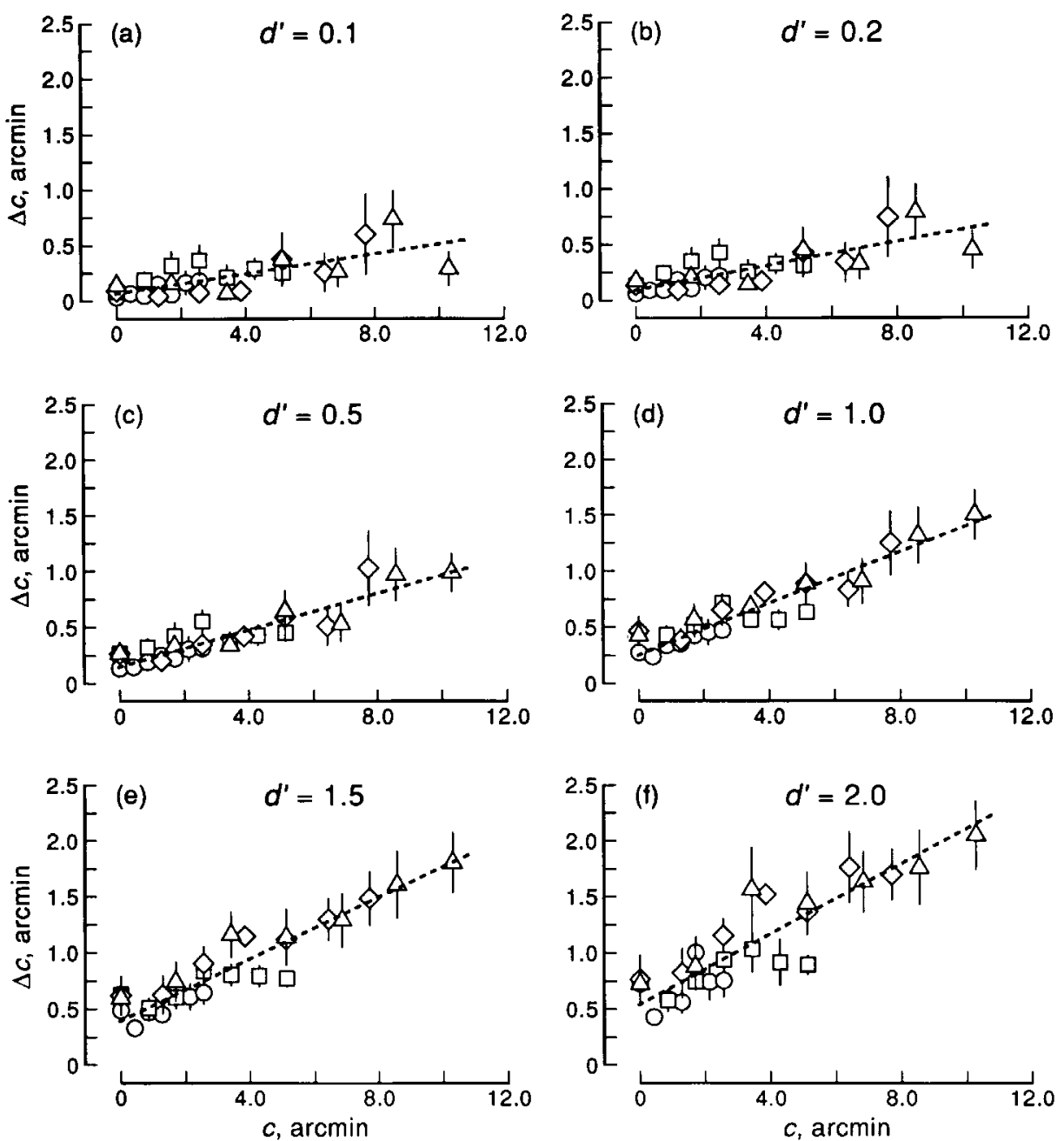

Figure 4. Sag increment-threshold functions for six criterion levels of discrimination performance defined by discrimination-index values $d^{\prime}$ of $0.1,0.2,0.5,1.0,1.5$, and 2.0 , corresponding to values of $53 \%, 56 \%, 64 \%, 76 \%, 86 \%$, and $92 \%$ correct, respectively. As in Fig. 3, the different symbols correspond to different enlargements of the curved lines, specified by their chord-lengths: $00.2 \mathrm{deg}$; $\square 0.4 \mathrm{deg} ; \diamond 0.6 \mathrm{deg}$; and $\triangle 0.8 \mathrm{deg}$. Each data point is the weighted mean over five subjects, and the vertical bars show $\pm 1 \mathrm{SEM}$. Broken lines are least-squares linear regressions (with the point $c=0$ arcmin omitted).

of performance of $75 \%$ correct, it was experimentally the most efficient cue of eight tested in increment-threshold measurements (Foster et al., 1993a; Foster and Wagemans, 1993). (3) A linear function of sag was sufficient to account for the variance in the increment-threshold data; that is, experimentally, Weber's law held almost exactly. (4) Efficiency and Weber's law were maintained despite changes in the criterion level of performance from $53 \%$ to $92 \%$ correct $\left(d^{\prime}=0.1\right.$ to 2.0 ).

In having these desirable theoretical and experimental properties for local contour-curvature description, sag need not of course be unique, and other 
equally efficient cues, including differential and semi-differential cues, might be contrived (Foster et al., 1993a,b). Sag also has some deficiencies, most notably the need for the contours to be finite (so endpoints are defined) and parallel (sag is only a relative invariant so long as the directions in which it is measured are parallel; Foster and Wagemans, 1993). The problem with infinite or extended contours is not insurmountable, for as has been previously noted (Foster et al., 1993a) sag might be defined with respect to points of inflexion in the curve; such points, in addition to being affine-invariant features of the contour, have been shown to be salient in a perceptual contour-segmentation task (Hanoulle et al., 1993); other invariant features for contour-segmentation have also been proposed (Watt, 1986). As to the problem of non-parallel contours, it is not known in detail how discriminability is impaired when curved lines have different orientations (Foster and Wagemans, 1993).

The fact that the statistical efficiency of sag was high over a range of criterion levels has other implications. In the aforementioned theoretical analysis of the relative invariance of some classical cues for contour-curvature discrimination (Foster and Wagemans, 1993), it was assumed that the viewpoint-invariance of an arbitrary ratio $c^{\prime} / c$ of cue values $c, c^{\prime}$ was equivalent to the invariance of a Weber fraction $\Delta c / c$, where $\Delta c=c^{\prime}-c$ for some criterion level of performance, which, in principle, could be set anywhere between $50 \%$ and $100 \%$ in a two-alternative forced-choice task. As noted earlier, only one criterion level, namely $75 \%$ correct, was used in practice (Foster et al., 1993a), but the results reported here show that this equivalence applies over criterion levels ranging from $53 \%$ to $92 \%$ correct. To further extend this range, however, more experimental trials would have to be performed at extreme stimulus levels. Even so, this partial equivalence suggests that something of the perceived relationships of shapes, which should in principle be definable over a broad range of values of the cue, may be usefully quantified-and in a largely viewpoint-invariant way-by Weber fractions specified for suitable criterion levels of performance.

This analysis has been essentially operational: no internal coding processes have been described that might compute these relative invariants that, in turn, produce observed behaviour. Nevertheless, the various mechanisms, operators, and systems for extracting invariants considered by Dodwell $(1957,1970,1992)$ may still be relevant, since invariants and relative invariants differ only by a scaling factor.

\section{Acknowledgements}

We are grateful to P. C. Dodwell, W. H. Mcllhagga, M. G. A. Thomson, and J. R. Wood for critical reading of the manuscript. This work was supported by the Medical Research Council and by EC Esprit Basic Research Actions No. 3001 (Insight) and No. 6448 (VIVA).

\section{NOTES}

1. A more general formulation of this viewpoint-invariance principle in terms of the perceived relationships of shapes was proposed in Foster and Wagemans (1993), where some additional motivational material drawn from the luminance domain was also included. 
2. For the transformed curves analysed here, the error in treating them as circular was small: deviations of each from a best-fitting circular arc did not exceed 3\% (Foster et al., 1993a).

3. Euclidean curvature (and radius of Euclidean curvature) measured at parallel tangents does, however, yield constant ratios (Foster et al., 1993b).

4. Nor indeed for Euclidean curvature measured at parallel tangents (Foster et al., 1993b).

\section{REFERENCES}

Aloimonos, J. Y. (1990). Perspective approximations. Image Vision Comput. 8, 179-192.

Attneave, F. (1954). Some informational aspects of visual perception. Psychol. Rev. 61, 183-193.

Cutting, J. E. (1986). Perception with an Eye for Motion. MIT Press, Cambridge, MA.

Dodwell, P. C. (1957). Shape recognition in rats. Br. J. Psychol. 48, 221-229.

Dodwell, P. C. (1970). Visual Pattern Recognition. Holt, Rinehart and Winston, New York.

Dodwell, P. C. (1992). Perspectives and transformations. Can. J. Psychol. 46, 510-538.

Efron, B. (1982). The Jackknife, the Bootstrap and Other Resampling Plans. CBMS-NSF Regional Conference Series in Applied Mathematics, No. 38; Society for Industrial and Applied Mathematics, Philadelphia, PA.

Falmagne, J.-C. (1985). Elements of Psychophysical Theory. Clarendon Press, Oxford.

Fisher, R. A. (1922). On the mathematical foundations of theoretical statistics. Phil. Trans. R. Soc. Lond. A222, 309-368.

Foster, D. H. (1977). Visual pattern recognition by assignment of invariant features and featurerelations. Optica Acta 24, 147-157.

Foster, D. H. and Bischof, W. F. (1991). Thresholds from psychometric functions: superiority of bootstrap to incremental and probit variance estimators. Psychol. Bull. 109, 152-159.

Foster, D. H. and Wagemans, J. (1993). Viewpoint-invariant Weber fractions and standard contourcurvature discrimination. Biol. Cybern. 70, 29-36.

Foster, D. H., Simmons, D. R. and Cook, M. J. (1993a). The cue for contour-curvature discrimination. Vision Res. 33, 329-341.

Foster, D. H., Wood, J. R., Van Gool, L. J., Wagemans, J., Pauwels, E. J. and Moons, T. (1993b). Differential affine invariants and contour-curvature discrimination. Perception 22 (suppl.), 11-12.

Green, D. M. and Swets, J. A. (1966). Signal Detection Theory and Psychophysics. Wiley, New York.

Hall, J. L. (1981). Hybrid adaptive procedure for estimation of psychometric functions. J. Acoust. Soc. Am. 69, 1763-1769.

Hanoulle, I., Waeytens, K., Wagemans, J., d'Ydewalle, G. and Van Rensbergen, J. (1993). Representation and segmentation of shape based on curvature singularities. Perception 22 (suppl.), 107.

Knill, D. C. (1992). Perception of surface contours and surface shape: from computation to psychophysics. J. Opt. Soc. Am. A9, 1449-1464.

Koenderink, J. J. and van Doorn, A. J. (1982). The shape of smooth objects and the way contours end. Perception 11, 129-137.

Kramer, D. and Fahle, M. (1993). On curvature detection in lines and edges. Invest. Ophthalmol. Visual Sci. 34 (Arvo Suppl.), 778.

Laming, D. (1986). Sensory Analysis. Academic Press, London.

Link, N. K. and Zucker, S. W. (1988). Corner detection in curvilinear dot grouping. Biol. Cybern. 59, 247-256.

Mundy, J. L. and Zisserman, A. (Eds) (1992). Geometric Invariance in Computer Vision. Cambridge, Massachusetts.

NAG (1987). The GLIM System. The Numerical Algorithms Group Limited, Oxford.

Niall, K. K. (1992). Projective invariance and the kinetic depth effect. Acta Psychol. 81, 127-168.

Reiss, T. H. (1993). Recognizing Planar Objects Using Invariant Image Features. Lecture Notes in Computer Science Series. Springer, Berlin.

Richards, W., Dawson, B. and Whittington, D. (1986). Encoding contour shape by curvature extrema. J. Opt. Soc. Am. A3, 1483-1491.

Stuart, A. and Ord, J. K. (1991). Kendall's Advanced Theory of Statistics, Volume 2: Classical Inference and Relationship, Fifth Edition. Edward Arnold, London.

Taylor, M. M. and Creelman, C. D. (1967). PEST: Efficient estimates on probability functions. $J$. Acoust. Soc. Am. 41, 782-787.

Watt, R. J. (1986). Feature-based image segmentation in human vision. Spatial Vision 1, 243-256. 
Watt, R. J. and Andrews, D. P. (1982). Contour curvature analysis: hyperacuities in the discrimination of detailed shape. Vision Res. 22, 449-460.

Weber, E. H. (1834). De Tactu. Koehler, Leipzig.

Winer, B. J. (1971). Statistical Principles in Experimental Design, Second Edition. McGraw-Hill, New York. 